\title{
SPECTRAL AND UBV DATA FOR 583 STARS \\ (SUPERGIANTS AND FOREGROUND STARS) \\ IN THE DIRECTION OF THE LARGE MAGELLANIC CLOUD
}

\author{
A. ARDEBERG, J. P. BRUNET, E. MAURICE, and L. PRÉVOT \\ European Southern Observatory, Santiago de Chile, and Observatoire de Marseille, France
}

\begin{abstract}
The stars observed are mainly from the Catalogue by Fehrenbach and Duflot. Some other stars - overexposed on the objective-prism plates, faint blue stars in $\mathrm{H}$ II regions, stars with overlapping objective-prism spectra - have been added.

The photoelectric $U B V$ photometry was done with the 1-m telescope at La Silla. The $U B V$ filters have always been those recommended by Johnson. Normally 3 measurements or more on different nights have been made for each star. For a single observation of a program star the internal standard deviations are
\end{abstract}

$$
\sigma_{V}=0.017, \quad \sigma_{(B-V)}=0.013, \quad \sigma_{(U-B)}=0.017
$$

The spectra have been taken with a Cassegrain spectrograph mounted on the 1.52-m telescope at La Silla. The dispersion is $73 \AA \mathrm{mm}^{-1}$, the spectral range $3250-5000 \AA$, and the limiting resolution $1.3 \AA$.

As a result of our observations, we present 409 stars as members of the LMC, 42 as possible members and 132 as galactic foreground stars (19 of these having high radial velocity). Remarkable features regarding velocities, spectra, magnitudes and colors are noted and commented.

All stars down to $B=11.5$ have been observed spectrographically as well as photometrically. This is also the case for the majority of the high-velocity stars down to $B=12.0$. For fainter objects the photometry is quite representative, whereas the spectroscopy is more scarce. The limiting magnitude is $B=14.0$.

As radial velocities were used as membership criterion, our data do not suffer from any spectral type selection effects.

It was easy to apply the MK criteria to our dispersion using the Yerkes atlas and the Bidelman list of supergiants. A continuous series of supergiant standards in the LMC has been established from O6 to G2. Comparison with Radcliffe data and with recent results of Divan gives a dispersion in spectral type of less than one tenth of a spectral class. Also the luminosity classification shows good agreement.

In the classification several difficulties were met:

(1) The LMC stars are very luminous objects. In the Galaxy we do not have many corresponding stars. This is especially so for later spectral types.

(2) With the dispersion used, the differences between $\mathrm{IaO}$ and Ia are not very pronounced, especially not for F-G type stars.

(3) Many of the LMC supergiants are P Cygni stars. They show very sharp, often 
asymmetric line profiles with a sharp rise to the long wavelength side. This might cause erroneous classification, and also affect radial velocities.

(4) The presence of an extended atmosphere, causing dilution effects, can give spurious spectral classification.

We have found $8 \mathrm{~A}$ type high-velocity stars. The $U B V$ results confirm their classification. 11 G-K type stars have been found to be high-velocity stars, among which are two subdwarfs earlier discussed by Fehrenbach and Thackeray. The number of late type high-velocity stars is quite high.

Full accounts have been given in Astron. Astrophys. Suppl. 6 (1972), 249. 